\title{
Correspondence analysis and categorical conjoint measurement ${ }^{\infty}$
}

\author{
Anna Torres-Lacombay
}

\begin{abstract}
A bstract
We show the equivalence between the use of correspondence analysis (CA) of concadenated tables and the application of a particular version of conjoint analysis called categorical conjoint measurement (CCM). The connection is established using canonical correlation (CC). The second part introduces the interaction exects in all three variants of the analysis and shows how to pass between the results of each analysis.
\end{abstract}

K ey words: Correspondence analysis, conjoint analysis, canonical correlation, categorical data.

J ournal of E conomic Literature classi..cation: C 19, C88.

\footnotetext{
${ }^{2}$ Requests for reprints should be sent to A nna Torres-Lacomba.

y $D$ epartment of E conomics and B usiness, U niversitat Pompeu Fabra, R amon Trias Fargas, 25-27, 08005 Barcelona (Spain). E-mail: anna.torres@econ.upf.es
} 


\section{Introduction}

Conjoint analysis is used in marketing and other ..elds to quantify individuals' trade-oxs when they can choose between multidimensional alternatives. Researchers ask the subjects to indicate their preferences for objects under a range of hypothetical situations. They use these judgements to estimate preference functions.

Conceptually, the researcher decomposes a respondent's overall preference judgements for objects de..ned on two or more attributes into part worths (partial utility values) for distinct attribute levels. With the resulting preference functions managers can predict the share of preference for any product under consideration, relative to other products. In other words, conjoint measurement procedures provide a methodological framework for the development of appropiate "psychophysical" transformations that can be used to ascertain the importance of classes of variables (e.g., color versus type of fragrance of soaps) as well as the scale values for various factor levels, (e.g. ‡oral, lemon, medicinal) (Green and Wind, 1972).

The method has been applied in virtually every conceivable product category, including consumer durables (e.g., automobiles), nondurables (e.g., soft drinks), industrial products (e.g., copiers), ...nancial services (e.g., checking accounts), and other services (e.g., hotel accommodation). Dixerent algorithms are used, according to the three forms of conjoint measurement: categorical, additive or simple polynomial (Wittink, 1999).

The technique of correspondence analysis, also used in marketing research and in many other ..elds, can be understood as a method for ..nding the association between the categories of two or more categorical variables and presenting this relationship in an easy visual format. This general de..nition has had different extensions that have led to the technique being applied in a wide range of situations involving dixerent types of categorical data, for example contingency tables, indicator matrices, preferences, paired comparisons and ratings.

The conjoint analysis process can be divided into four phases: data collection, measurement scale for respondent judgments, parameter estimation methods and market simulation. The present work is concerned with the parameter estimation method, or the third step in the process of a conjoint analysis. For the particular case of full pro..le collection method and rating scale in the measurement of respondent judgments, the nonmetric method used to analyze the data is known as Categorical Conjoint M easurement (CCM ). Our objective is to show the equivalence between the CCM algorithm due to Carroll (1969) and a particular case of correspondence analysis (CA ). Once the equivalence is shown, we try to see if the equivalence is maintained when interaction exects are included. Green and Wind (1972) say that one extension of CCM would be to include the interaction exects. The same idea is noted in Green (1973). 
We shall start by describing the objective and the results of a CCM analysis followed by a brief introduction of canonical correlation analysis (CC). It is useful as an intermediate stage, since the equivalence between CCM and CC is already shown (Carroll, 1969). The equivalence between CC and CA has been shown for the particular case where there is one attribute being related to preference (see, for example, G reenacre 1984, chap.4). We will see what happens when two or more attributes are being related to preference and ..nally we will compare the results obtained from the analysis of the data using CCM , CC and CA. Later on, we will introduce the way to code the data so that CA can treat interactions exects. We will repeat the operation with CC as well as with CCM to demonstrate the equivalence empirically.

The..rst data set is from the paper of Rao (1977). It comes from the situation of an apartment-dweller planning to purchase a house that is already built in a college town. The decision-maker has isolated the attributes of the house considered most important in the decision. The attributes are three: size of the house ( 3 levels), price of the house ( 4 levels) and general condition of the house ( 3 levels) and the response variable has 4 levels. The second data set is from an airline company. The objective in this case is to know the trade-or value between the dixerent attributes oxered as well as possible interaction exects between them. The attributes are: airline company ( 5 levels), price ( 5 levels), service ( 3 levels) and timetable ( 3 levels). The response variable has 4 levels.

\section{M ethods}

\subsection{Categorical conjoint measurement (CCM)}

We are interested in the analysis of a matrix of dummy variables, called an indicator matrix, of the following form:

2

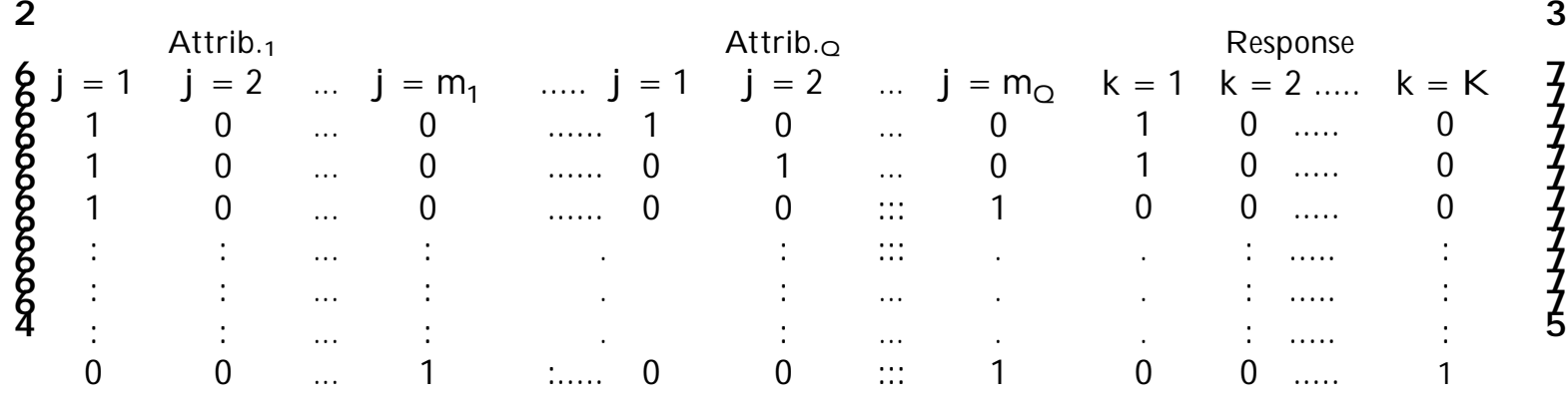

where

$\mathrm{m}_{\mathrm{q}}$ : number of levels for the attributes $\mathrm{q}=1 ; \ldots ;: \mathrm{Q}$.

$\mathrm{K}$ : number of response categories where $\mathrm{k}=1 ;: ;: ; \mathrm{K}$ : 

levels.

$M, \mathrm{Q}_{\mathrm{q}=1} \mathrm{~m}_{\mathrm{q}}$ : number of all the possible combinations of the attribute

The above indicator matrix is made up of a matrix $Z_{1}$ of dummy variables representing the full pro..le (i.e., the indicator matrix has $M$ rows) and another matrix $Z_{2}$ of dummy variables indicating one subject's preferences for each combination. For the additive case, the objective is to ..nd an optimal additive combination of scale values for the attribute levels $Z_{1}$ a maximally correlated with the response categories assigned to each combination, $Z_{2} b$.

Here $\underline{b}$ is the vector that collects the optimal scale values for the category $\mathrm{k}$ of the response variable, and $\underline{a}$ is the vector with the optimal scores for all ${ }_{q} m_{q}$ levels of the attributes.

Carroll (1969) has shown that this analysis is equivalent to canonical correlation analysis of the dummy variables. He also shows the equivalence between applying canonical correlation and the following formulation which is the one we are going to use to show the equivalence between CCM and CA .

First step De..ne:

$$
s_{q ; j ;} ; k^{\prime} \frac{r}{\frac{m_{q}}{n_{k} M}}\left(n_{q ; j ; ; k} i \frac{n_{k}}{m_{q}}\right)
$$

where: $\mathrm{j}_{\mathrm{q}}^{\text {th }}$ level of attribute $\mathrm{q}$ :

$\mathrm{n}_{\mathrm{q} ; j_{\mathrm{j}} ; \mathrm{k}^{\prime}}$ number of times $\mathrm{k}^{\text {th }}$ response category value occurs in $(\mathrm{k}=1 ;:: ; \mathrm{K})$ :

$n_{k}$ ' total number of times $k^{\text {th }}$ response category value occurs

Second step Let $S_{q}$ be the $m_{q} £ K$ matrix whose general entry is $S_{q ; j} ; k$. De..ne the $K £ K$ matrix $R$ as:

$$
R={ }_{q=1}^{Q} S_{q}^{\top} S_{q}
$$

Third step Determine the eigenvectors $v_{l}$, for each dimension, of $R$ and then the optimal scores $\mathrm{w}_{\mathrm{I}}$ as:

$$
w_{1}=p_{\overline{n_{k}}}^{v_{1}}
$$

Notice that each attribute is initially treated separately, then combined in the matrix $\mathrm{R}$, which is decomposed in order to ..nd scores for the response categories only. 
The data set used is from $R$ ao (1977), and has three attributes $(Q=3)$ : 4.

1: Size of house (number of bedrooms), where $m_{1}=3$, with levels 2,3 and

2: A sking price (thousands of dollars), where $m_{2}=4$; with levels $25,30,35$ and 40.

3: Condition of the house, where $m_{3}=3$; with levels $E$ : Excellent, $G$ : Good and $\mathrm{P}$ : Poor.

The number of attribute combinations is $M=3 £ 4 f 3=36$. The response has $K=4$ categories: $A$ : very high worth; $B$ : just high worth; $C$ : just low worth; D: very low worth. The data are given in appendix I.

\subsection{Correspondence analysis (CA)}

In the simple CA of a two-way table, we are interested in explaining the association between the row and column categories, representing the association in a low-dimensional space in the form of a map. The overall association is quanti..ed by the chi-squared statistic divided by $n_{++}$(the total number of cases), i.e. $\hat{A}^{2}=n_{++}$; called total inertia:

$$
\frac{\hat{A}^{2}}{n_{++}}={\frac{1}{n_{++}}}_{j=1 k=1}^{X} \frac{\left(n_{j k} i n_{j+} n_{+k}=n_{++}\right)^{2}}{\left(n_{j}+n_{+k}=n_{++}\right)}
$$

where $n_{j k}$ is the number of cases in a particular cell, $n_{j}+$ the row total, $n_{+k}$ the column total and $\mathrm{n}_{++}$is the grand total.

The decomposition of this objective function is analogous to ..nding the largest principal component of a set of $\mathrm{J}$ observations on $\mathrm{K}$ variables with the generalization to accommodate dixerent weights, called masses. If we analyze the row pro..le matrix, where each row of the table is divided by its total, the row masses $r$, are the row totals divided by the grand total. Column pro..les and the column masses $c$ are similarly de..ned. The row pro..les have centroid equal to $c$ and the column pro..les have centroid $r$ :

The row and column coordinates of the pro..les with respect to their respective principal axes may be obtained from the singular value decomposition (SVD) of the matrix $N=\left(n_{j k}\right)$, transformed by double-centring and standaridzing:

$$
D_{r}^{i \frac{1}{2}}\left[\left(1=n_{++}\right) N \text { i rc } c^{\top}\right] D_{c}^{i \frac{1}{2}}=U D_{\circledast} V^{\top} ;
$$

where $U^{\top} U=V^{\top} V=I$ : The singular values are the square roots of the principal inertias or eigenvalues: $D_{\circledast}=D^{\frac{1}{2}}$ and $D_{r}$ and $D_{C}$ are diagonal matrices with the row and column masses, respectively, in their main diagonal. 
The principal axes of the row and column pro..les are the column vectors of $D_{r}^{\frac{1}{2}} V$ and $D_{c}^{\frac{1}{2}} U$; respectively. For example, the two-dimensional coordinates of pro..le points (i.e., from the row pro..le matrix, each row becomes a point to be represented in the map) and vertex points (i.e., each column is a vertex point for the row pro..les points) in the dual problems are the rows of the ..rst two columns of the following matrices:

Row problem : row pro..les, $D_{r}^{i \frac{1}{2}} U D_{\circledast} ;$ column vertices, $D_{C}^{i \frac{1}{2}} V$ : The rows will be the points projected in a map interpreted in terms of the columns, which have contributed the most to the orientation of the principal axes.

Column problem: column pro..les, $D_{c}^{i \frac{1}{2}} V D_{\circledast}$; row vertices, $D_{r}^{i}{ }^{\frac{1}{2}} U$ : The colums will be the points projected in a map interpreted in terms of the rows, which have contributed the most to the orientation of the principal axes.

\subsection{Canonical correlation (CC).}

The geometry of canonical correlation is given by Greenacre, (1984, section 4.4) and also its relationship to the geometry of the correspondence analysis of an indicator matrix, for the classical case where two categorical variables are treated. As we noted before, since categorical conjoint analysis can be applied to more than two attributes, the equivalence between this technique and the correspondence analysis of an indicator matrix, via canonical correlation, is not obvious. We will describe the basic geometry of CC and we will introduce the new de..nitions and operations that will let us establish the connection.

The objective of CC is to ..nd strong linear relationships between two sets of variables (two-way table in CA) as observed across the sample of $n_{++}$cases. If $Z_{1}$ and $Z_{2}$ are the data matrices corresponding to the two sets of variables, this objective can be expressed formally as ..nding linear combinations $Z_{1} a$ and $\mathrm{Z}_{2} \mathrm{~b}$, which have maximum correlation $1 / 2$

$$
\left.1 / 2=\left(a^{\top} S_{12} b\right)=\left(a^{\top} S_{11} a\right)\left(b^{\top} S_{22} b\right)\right)^{\frac{1}{2}}
$$

where $S_{12 ;} S_{11}$ and $S_{22}$ are the covariance matrix between $Z_{1}$ and $Z_{2}$ and the covariance matrices of $Z_{1}$ and $Z_{2}$ respectively.

The vectors $a_{k}$ and $b_{k}$ of canonical weights can be obtained from the left and right singular vectors of the matrix $S_{11}^{i \frac{1}{2}} S_{12} S_{22}^{i} \frac{1}{\frac{1}{2}}$ (See for example, G reenacre 1984): The SVD of the matrix is:

$$
\mathrm{S}_{11}^{\mathrm{i} \frac{1}{2}} \mathrm{~S}_{12} \mathrm{~S}_{22}^{\mathrm{i} \frac{1}{2}}=\mathrm{UD}_{1 / 2} \mathrm{~V}^{\top} \text { with } \mathrm{U}^{\top} \mathrm{U}=\mathrm{V}^{\top} \mathrm{V}=\mathrm{I} \text { : }
$$

where $D_{1 / 2}$ is a diagonal matrix with the canonical correlations in the diagonal, $\mathrm{U}$ and $\mathrm{V}$ are the left and right singular vectors. 
The matrices of canonical weights are:

$$
A=S_{11}^{i \frac{1}{2}} U \text { and } B=S_{22}^{i \frac{1}{2}} V
$$

The standarization of the singular vectors of $\mathrm{U}$ and $\mathrm{V}$ to be orthonormal as in (8) implies that $A$ and $B$ are standarized as follows:

$$
A^{\top} S_{11} A=B^{\top} S_{22} B=1
$$

The usual standarization of the vectors of canonical scores is that all of them have unit variance. At the same time it is also a set of identi..cation conditions on the scale of the canonical weights and of the canonical scores. In order to identify the origins of the vectors of canonical scores, their means are conventionally set at zero, which is equivalent to each variable of $Z_{1}$ and $Z_{2}$ being centered with respect to its mean.

In this particular application, the ..rst set of variables consists of 10 dummy variables for the attribute levels and the second set consists of 4 dummy variables for the response categories. The rows are the 36 possible combinations of attribute levels. Thus $Z_{1}$ and $Z_{2}$ have the following form:

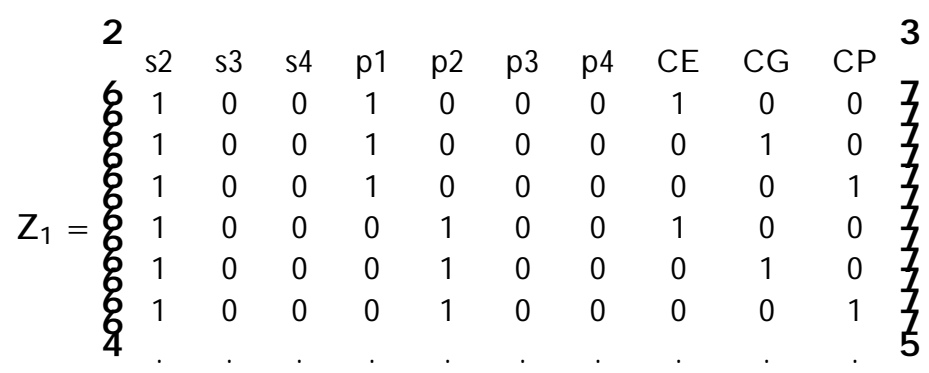

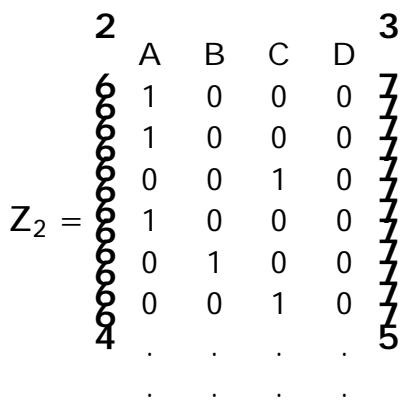

For example, the ..rst combination of attributes, two bedrooms in the house, a price of $\$ 25.000$ and excellent condition, gets a response of "very high worth". When an indicator matrix is analyzed, $S_{11}$ and $S_{22}$ are singular matrices, which implies that they cannot be inverted. To be able to do our computations, the last level for each attribute and the last response category will be eliminated. This operation lets us to estimate the canonical weights. 


\subsection{CA of a concatenated table and its connection with CC.}

The operation of eliminating the last level for each attribute and the last category, lets us estimate the canonical weights.

The ..rst important dixerence with respect to Greenacre (1984, section 4) is the data matrix to be analyzed. In this case, the matrix $Z_{1}^{\top} Z_{2}$ is a concatenated table, whereas in previous work, $Q=1$; that is there is only one categorical variable in the ..rst set and then $Z_{1}^{\top} Z_{2}$ is a single crosstabulation: for this simpler case Greenacre (1984) shows how the CA results can be obtained from the canonical weights by imposing the centring conditions of $C A$, using the masses in the weighted averaging procedure. In the present case where $\mathrm{Q}>1$, we again impose the CA condition to recover the standard coordinates for the categories and the principal coordinates for the levels of the dixerent attributes. $F$ inally we check that the coordinates obtained are identical to the ones obtained in the correspondence analysis of the concatenated table $T{ }^{\prime} Z_{1}^{\top} Z_{2}$ :

Canonical correlation analysis gives solutions, for each axis, of the form:

Attributes:

$a_{q}^{\alpha}=\left[a_{q ; 1}^{\alpha}: \cdots: a_{q ; m_{q i}}^{\alpha} 0\right]^{\top}$ where $q=1 ;: \ldots:: ; Q$

Response:

$b^{\alpha}=\left[b_{1}^{\alpha}::: b_{k i 1}^{\alpha} 0\right]^{\top}$

The correspondence analysis results are obtained from these, as follows (for each dimension):

Attributes:

$q=1 ;:::: ; Q$

$$
a_{q ; m_{q}}=\sum_{j=1}^{n_{* i} i^{1}} r_{q ; j} a_{q ; j q}^{\alpha} \quad a_{q ; j q}=a_{q ; j_{q}}^{\alpha}+a_{q ; m_{q}} \quad j=1 ;: ;: ; m_{q}
$$

Response:

$$
b_{k}=\sum_{k=1}^{k_{i}{ }^{1}} c_{k} b_{k}^{\alpha} \quad b_{k}=b_{k}^{\alpha}+b_{k} \quad k=1 ;: ;: ;: ; k
$$

\subsection{Results}

From the CC numerical solution, which appears in appendix I, we recover the values of the standard coordinates of the categories in CA by applying the above transformation.

For example, for the ..rst dimension, we obtain:

$$
9\left(2: 9321+b_{k}\right)+12\left(2: 0246+b_{k}\right)+9\left(1: 1488+b_{k}\right)+6 b_{k}=0
$$




$$
b_{k}=i \text { 1:6951 }
$$

Then,

$$
\begin{aligned}
& b_{1}=1: 237 \\
& b_{2}=0: 3295 \\
& b_{3}=i 0: 5463 \\
& b_{4}=i 1: 6951
\end{aligned}
$$

This operation is repeated for the second and the third principal axes, as well as all the process for the attributes.

To corroborate the results, we ran the SimCA program (Greenacre, 1986) to get the CA principal coordinates and then converted these to the following standard coordinates (see appendix I):

\begin{tabular}{|cccc|}
\hline & Dim: 1 & Dim: 2 & Dim: 3 \\
A & $1: 2545$ & $0: 7888$ & $0: 8966$ \\
B & $0: 3342$ & $1: 3029$ & i $0: 4367$ \\
C & i $0: 5540$ & $1: 0485$ & i $1: 2624$ \\
D & i $1: 7191$ & i $0: 1500$ & $1: 4220$ \\
\hline
\end{tabular}

$\mathrm{q}$ The values agree with those recovered from CC once the correction factor $\frac{\mathrm{M}_{\mathrm{j}} 1}{\mathrm{M}}=\frac{35}{36}$ is applied. This is due to the computation of unbiased variances in $\mathrm{CC}$.

\section{Equivalence of CCM and CA}

So far, we have established the relationship between CA of concatenated tables and canonical correlation analysis where one set of variables is composed by several categorical variables. We now look at the relationship between Carroll's CCM and CA, showing that there are simple scaling factor dixerences in the eigenvalues (principal inertias in CA) and the response category scores (standard coordinates in CA). We show these relationships by detailing the CCM and CA theory side by side.

\subsection{R elation between the eigenvalues}

First Step

$$
\begin{aligned}
& \text { Categorical Conjoint M easurement } \\
& s_{q ; j_{q} ; k}, \frac{r}{\frac{m_{q}}{n_{k} M}}\left(n_{q ; j_{q} ; k} i \frac{n_{k}}{m_{q}}\right)
\end{aligned}
$$


where $\mathrm{k}$ is the response category assigned to the level $\mathrm{j}_{\mathrm{q}}$ of the factor $\mathrm{q}$ :

\section{Correspondence Analysis.}

From (5) the centered and standarized matrix can be written as:

$$
\begin{aligned}
& t_{q ; j ; k}=\frac{\left(\frac{n_{q ; j} ; k}{Q M} i \frac{1}{Q m_{p}} f \frac{n_{k}}{M}\right)}{\overline{\frac{1}{Q m_{q}}} f \overline{\frac{n_{k}}{M}}}= \\
& \frac{1}{Q M} \pm \frac{{ }^{P}{ }_{Q^{p}}^{p}{ }_{m_{q}}^{p} \bar{M}}{P_{\overline{n_{k}}}} f\left(n_{q ; j ;} ; k i \frac{n_{k}}{m_{q}}\right)=
\end{aligned}
$$

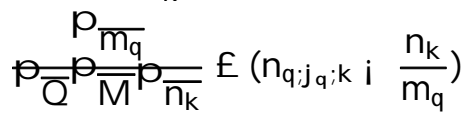

$$
\begin{aligned}
& t_{q ; j ; j}=p_{\bar{Q}}^{1} f \frac{r}{\overline{m_{q}}} f\left(n_{q ; j} ; k i \frac{n_{k}}{m_{q}}\right)
\end{aligned}
$$

Thus, there is only a scaling factor equal to $\mathrm{P}_{\overline{\mathrm{Q}}}^{1}$; linking the two approaches, where $\mathrm{Q}$ is the number of attributes.

Second Step From the previous operations we obtain $S_{q ; j} ; k$ as well as $T_{q ; j} ; k$ which corresponds to the CCM and CA matrices that collect the centered and standarized data for each factor, for each level and for each one of the categories, calculated previously. Then we follow with the operation,

$$
\begin{array}{cc}
\mathrm{CCM} & \mathrm{CA} \\
\mathrm{R}=\mathrm{q}_{\mathrm{q}=1}^{\mathrm{Q}} \mathrm{S}_{\mathrm{q}}^{\top} \mathrm{S}_{\mathrm{q}} & \mathrm{T}^{\top} \mathrm{T}=\frac{1}{\mathrm{Q}} \underset{\mathrm{q}=1}{\mathrm{P}} \mathrm{T}_{\mathrm{q}}^{\mathrm{T}} \mathrm{T}_{\mathrm{q}}=\frac{1}{\mathrm{Q}} \mathrm{R}:
\end{array}
$$

which gives the relationship

$$
\frac{1}{\mathrm{Q}}, \mathrm{CCM}=, \mathrm{CA}
$$

where, CCM and, CA are the eigenvalues obtained applying CCM and CA respectively. This relation is corroborated with the data set. Thus the principal inertias in CA are equal to the eigenvalues from CCM divided by Q.

Since the CA of a concatenated table is the average of the inertias of the individual tables (Greenacre, 1994), the total variance in CCM is just the sum of the inertias of the $\mathrm{Q}$ tables. 


\subsection{R elationship between the coordinates of CCM and CA}

We use the third step in Section 2.1 and the CA theory in Section 2.2. The standard coordinates in CA are de..ned as

$$
Y=D_{c}^{i \frac{1}{2}} V
$$

where $Y$ are the column standard coordinates, $D_{c}$ are the diagonal matrix with the column masses in the main diagonal and $\mathrm{V}$ are the right singular vectors. Since $c_{k}=\frac{n_{k}}{M}$ it follows from (12) that:

$$
\begin{gathered}
y_{l}=\frac{p_{\bar{M}}}{\overline{n_{k}}} v_{l} \\
p_{\overline{n_{k}}} \frac{p_{\bar{M}}}{\bar{M}}=v_{l}
\end{gathered}
$$

Hence from (3), $p_{\bar{M}}^{y_{1}}$ are the response category scores in CCM.

Thus the response category scores obtained by CCM are the same as the standard coordinates obtained when we apply CA to the concatenated table but rescaled by the factor $\mathrm{p} \frac{1}{\bar{M}}$ :

\subsection{Results}

For the ..rst dimension, the values are the following:

\begin{tabular}{|ccc|}
\hline & CA standard coordinates & CCM estimated coed cients \\
A & $1: 2545$ & i $0: 2091$ \\
B & $0: 3342$ & i $0: 0557$ \\
C & i $0: 5540$ & $0: 0923$ \\
D & i $1: 7191$ & $0: 2865$ \\
\hline
\end{tabular}

where the relation is equal to $p_{\overline{36}}^{1}$ :

The relation between the eigenvalues is the following:

\begin{tabular}{|ccc|}
\hline Eigenvalues & CA & CCM \\
.1 & $0: 4755$ & $0: 9501$ \\
.2 & $0: 2583$ & $0: 5166$ \\
.3 & $0: 0440$ & $0: 0880$ \\
\hline
\end{tabular}

In this case $\mathrm{Q}=2$ since the variable "size" was not included in the analysis because it has zero inertia. We corroborate that the two sets of eigenvalues dixer by a factor of 2 : 


\section{Interaction Exects}

A s noted previously, Green and W ind (1973) have pointed out as future research the possibility to introduce interaction terms explicitly in categorical conjoint measurement. The same idea is reł ected once again in G reen (1973).

We now show how CA is able to handle interaction exects. Furthermore, we show the connection again with the analysis of interactions using CC and CCM .

We illustrate our approach using a study designed by an airline company. At the beginning, the interest of the study was to examine if a particular respondent, with a particular pro..le, had dixerent perceptions between dixerent airline companies, dixerent prices, service levels and timetables. The four attributes are the following:

1. Airline company, where $m_{1}=5$; with levels: TWA, IBERIA, KLM, $B$ ritish A irways and TAP.

2. $P$ rice, where $m_{2}=5$; with levels (in pesetas): Levels: $P 1=85.000, P 2=$ $100.000, P 3=115.000, P 4=130.000$, and P $5=145.000$.

3. Service, where $\mathrm{m}_{3}=3$; with levels: $\mathrm{S} 1=$ bellow the mean, $\mathrm{S} 2=$ in the mean and $\mathrm{S} 3=$ above the mean.

4. Timetable, where $m_{4}=3$; with levels: $T 1=3$ hours before of your preferences, $\mathrm{T} 2=2$ hours before and $\mathrm{T} 3=1$ hour before.

\subsection{Correspondence analysis. R esults.}

\subsubsection{A nalysis without interaction exects}

In this particular case, we have $5 £ 5 £ 3 £ 3=225$ combinations. The analysis is applied at the individual-response level, getting the utility model for this person's pro..le. We use the answers from one of the PhD students in the Universitat Pompeu Fabra (B arcelona, Spain) as an illustration of the approach.

The output of the CA appears in appendix II. As shown in previous sections, the data are coded as a concatenated table, obtaining the same results as a conjoint analysis which has been suitably rescaled. The interpretation of the map is the following (see ..gure 1 in appendix II):

Prices:

The ..rst principal axis contains $70: 8 \%$ of the total information (total inertia $=0: 3123$ ), and the ..rst two principal axes contain 96:2\%. This big concentration of variance in the ..rst principal axis is because of the type of data we are analyzing. The response category points form a curve known as the "horseshoe" or "arch" which is common for data on an ordinal scale. O ne extreme of the ..rst principal axis will indicate high levels of utility and the other extreme, low levels of utility. The second principal axis normally dixerenciates the attribute levels depending on the distribution in the intermediate levels. Given the meaning in terms of utility of the principal axes, we can ...nd that the cheapest prices, $P_{1}$ and $P_{2}$, very correlated with the ..rst principal axis, are situated near the category which represents the higher utility level. On the other hand, while $\mathrm{P}_{3}$ 
is highly correlated with the second principal axis and near the high utility $B$, $\mathrm{P}_{4}$ and $\mathrm{P}_{5}$, correlated also with the ..rst principal axes, are situated near the lowest level of utility, D.

\section{Companies:}

TWA, KLM and B.A. are all associated with high levels of utility. The common characteristics among them are that they are non-national and have a well-known reputation. TAP is the one that is most disliked, maybe because it is not well known in Spain. IBERIA is dixerenciated with respect to the others. It has an important correlation with the second principal axis because of the mixture of answers in the extreme categories. This is the only national company.

\section{Services:}

The extreme levels, $S_{1}$ and $S_{3}$, are more identi..ed with the ..rst principal axis. The level representing "a service above the mean" is rated the best and the one which represents "a service below the mean" the worst rated. The intermediate level, $\mathrm{S}_{2}$, has a strong correlation with the second principal axis, given a more equalized distribution of the answers between the dixerent category levels.

\section{Timetable:}

W ith a similar pattern as the service attribute, the extreme levels, $T_{1}$ and $T_{3}$, have a high correlation with the ..rst principal axis and so they are associated with the extreme utility category levels. The dixerence with respect to the attribute of service is that the attribute level $\mathrm{T}_{2}$; which refects the mean level, totally explained by the second principal axis, is situated above the ..rst principal axis because of a bigger concentration of answers in the extreme categories.

At this point the analysis oxers, for a particular subject, the utility of each attribute. But we do not stop here since further interests come with the following idea. W hen a subject has to evaluate attributes referring to a long $¥$ ight it may be possible that a combination of two variables generates a utility signi..catively bigger or smaller that the sum of the utilities previously obtained. In applied terms this can be translated as the possibility to sell special oxers that could contain the lower level of price with adjustments in other attributes like the service level (lower value) or the timetable (quite far from the preferences) or even changing the airline company. These considerations imply perceptions that are dixerent from the linear combination of utilities previously estimated. Since "price" is the variable with bigger inertia in the previous analysis, we constructed an interaction variable composed of "price" and another variable. The dixerent levels of "airline company" have similar utilities, since in terms of signals of quality, all of the companies are quite homogeneous. The exception is TA P, and the reason can be the ignorance. The levels of "service" have small inertia as well as their interactions with the rest of variables. The reason can be a small real dixerence between the levels of this variable. But the situation 
is dixerent with the "timetable" attribute. The total inertia increases more and the new results are adding interesting information (see appendix II).

\subsubsection{Introduction of interaction exects}

The new variable, "price $£$ timetable" has 15 levels, which can be labelled as: $\mathrm{PT}_{11}, \mathrm{PT}_{12}, \mathrm{PT}_{13}, \ldots, \mathrm{PT}_{51}, \mathrm{PT}_{52}, \mathrm{PT}_{53}$, where the letters indicate the original variables and the subindices indicate the levels of the two attributes.

The data matrix to be analyzed and the results appear in appendix II. Once again we code the data as a concatenated table. It includes three active variables with their levels: the interaction variable (price $f$ timetable), airline company and service. Further, the original variables, price and timetable, will be added as supplementary points, being the centroids of the interactions. The reason is not to repeat information. The interaction variables include the within (eg., the utlilty of $P T_{1 j_{2}}$ versus the one of $P T_{1 j_{2}^{0}}$. The price label is the same in both variables, but the timetable levels are dixerent: $j_{2} \in j_{2}^{0}$ ) and the between inertia (eg., $P T_{1 j_{2}}$ and $P T_{2 j_{2}^{0}}$ where dixerent levels for both attributes are taken) while the main variables contribute only with the betwen inertia.

We are going to compare the new inertia and the one previously obtained, to be able to quantify its increases and hence justify the inclusion of the interaction terms:

$$
\begin{array}{lc}
\text { Inertia without interactions: } & \text { Between }=0: 3123 \\
\text { Inertia with interactions: } & \text { B etween }+W \text { ithin }=0: 6188
\end{array}
$$

With the introduction of the interaction exects, we double the total inertia. We can interpret the results from the map that appears in appendix II (see ..gure 2). The ..rst principal axis still dixerenciates between the most and the least preferred levels. The original levels (supplementary points) appear as the centroids of the interactions. The interactions show us that for the lowest level of the factor price, the variable timetable does not matter, in other words, if the price is the cheapest one, it does not matter if the timetable of the $¥$ ight is not adjusted to your preferences. The reason can be the fact that we are treating transatlantic ¥ights where the attribute timetable is less important than for shorter business travel where you might need to depart and return in the same day. On the other hand, if the price is the highest one, you only accept the timetable more adjusted to your preferences. We can see that once prices increase, the timetable attribute takes exect again, in such a way that to mantain the same utility, a timetable more adjusted to ones preferences is required. These trade-ox exects are collected on the second principal axis. We can conclude that as price increases, the interaction levels more correlated with the second principal axis refect the timetable level you require to compensate the increase in price and to stay with an intermediate utility, B or C, and not going to the $D$ one. 
Finally we calculate the standard coordinates for the categories. Later we will compare them with the ones obtained in $\mathrm{CC}$ to check the equivalence.

\begin{tabular}{|rrrr|}
\hline & Dim:1 & Dim:2 & Dim:3 \\
A & $1: 242$ & $0: 849$ & i $0: 506$ \\
B & $0: 427$ & i $0: 990$ & $1: 361$ \\
C & i $0: 520$ & i $1: 347$ & i $1: 553$ \\
D & i $1: 283$ & $0: 881$ & $0: 308$ \\
\hline
\end{tabular}

\subsection{Canonical Correlation}

When we include interaction exects, the relation between $C A$ and $C C$ is less obvious than in the previous case. We need one step more to understand the previous equivalence. The scheme of the explanation will be the following. First of all, we will describe the way to code the interaction in CC as a single dummy variable, in order to obtain identical results to those of CA. Secondly, we will show the equivalence between the results obtained with this approach and the results obtained with the more customary way of handling categorical variable interactions in linear models.

When we introduce an interaction variable, the type of data matrix to analyze in CA is still a concatenated table, in this case composed of 3 variables, one with 15 levels (pricef timetable) and the others with 5 levels (company) and 3 levels (service). It suggests immediately that CC has to be computed as before and that we have to omit one of the PT levels, one of the company levels and one of the service levels. We drop the last level of each one. Finally we recover the $\mathrm{CA}$ results just as before.

We point out that the usual way of handling categorical variables plus their interactions in linear models would be to drop one category of each one of the main exects and all categories of the interactions involving these levels (see the example in the appendix II). In this case, the problem is how to be able to recover all the coec cients, especially those of the interaction terms which are not so obvious. The key point to realize is that even though this way of centering in the old way could bring to us to the conclusion that each interaction set is treated as a dixerent variable, it is not like this in the calculations. All the interactions belong to the same variable and so all the omitted coec cients take the same value.

Once we consider the previous explanation, since the results should be the same, we know that the new way collects in its coec cients both main and dixerential exects, which should be obtained separately in the traditional way.

\subsubsection{Operation of centering. New way.}

We have 2 attributes composing the new interaction variable, where $\mathrm{j}_{1}=$ $1 ;:: ; ; m_{1}$ and $j_{2}=1 ;:: ; m_{2}$ are the levels for each attribute. To make the 
notation slilghtly easier and since we could be studying the interaction of any pair of variables, we set: $j_{1}{ }^{\prime} j, j_{2}{ }^{\prime} j^{0}$ and $m_{1}{ }^{\prime} m, m_{2}=m^{0}$ : The total number of levels for the interaction variable is equal to $\mathrm{m} f \mathrm{~m}^{0}$ : The operation of centering to recover the coec cients will be the following,

$$
d_{m m^{0}}={ }_{j j^{0} \in m m^{0}}^{X} r_{j j} d_{j j^{0}}^{\alpha}
$$

We consider all the coec cients, which correspond to all the possible interaction combinations, except the one composed of the last level of each attribute. The mass $r_{j j}{ }^{0}$, is in this case the total number of cases for a particular interaction level, which is composed of the levels $j$ for the ..rst attribute and $j^{0}$ for the second, and $d_{j j}^{a}$ is the coec cient obtained from the $C C$ estimation in the new way, corresponding to level $\mathrm{j}^{0}$ of the interaction variable. Since in this case, $r_{j j}{ }^{0}=\frac{1}{m f m^{0}}$ for all $j^{0}$,

$$
\begin{aligned}
& \frac{1}{m f m^{0}}{ }^{X} X\left(d_{j j}^{\alpha}{ }^{0} \in m_{m m^{0}}\right)+\frac{1}{m f m^{0}} d_{m m^{0}}=0 \\
& { }^{j j}{ }^{0} \in \stackrel{m}{X}^{0} \\
& \text { jje }{ }^{0} \in \operatorname{mx}^{0} \\
& \left(d_{j j}^{\alpha}+d_{m m^{0}}\right)+d_{m m^{0}}=0
\end{aligned}
$$

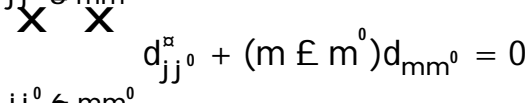

$$
\begin{aligned}
& \mathrm{jj}^{0} \in \mathrm{mm}^{0} \quad \mathrm{P} P \\
& d_{m m^{0}}=\frac{j^{j} j^{0} \epsilon m m^{0} d_{j j}^{d o}}{m f m^{0}}
\end{aligned}
$$

From here, we recover the coec cients as before:

$$
d_{j j} 0=d_{j j}^{\alpha}+d_{m m^{0}}
$$

\subsubsection{Operation of centering. Traditional way.}

$$
\min _{j=1 j^{0}=1}^{1 n^{0} i^{1}} r_{j j^{0}}\left(e_{j j^{0}}^{\alpha}+c\right)+\frac{1}{m f m^{0}}\left(m_{i} 1+m^{0}\right) c=0
$$

where $e_{j j^{\circ}}$ is the coed cient obtained from the CC estimation in the traditional way, corresponding to the level of the interaction variable composed of the levels $\mathrm{j}$ for the ...rst attribute and $\mathrm{j}{ }^{0}$ for the second attribute and $\mathrm{c}$ is the estimated coec cient corresponding to the interaction levels: $(\mathrm{ml}) ;(\mathrm{m} 2) ; \ldots: \ldots ;\left(1 \mathrm{~m}^{0}\right) ;\left(2 \mathrm{~m}^{0}\right) ; \ldots: .$. 
Since $r_{j j^{0}}=\frac{1}{m f m^{0}}$;

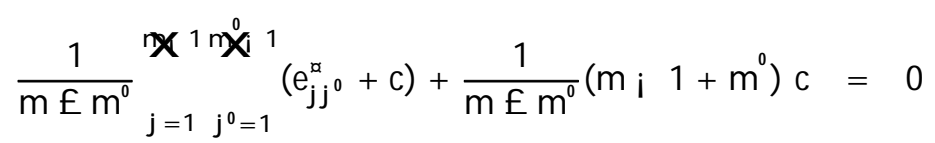

$$
\begin{aligned}
& \left(m_{i} 1\right)\left(m^{0} i 1\right) c+x_{j=1 j^{0}=1}^{x_{i} 1 n^{0} i 1} e_{j j^{0}}^{x}+\left(m_{i} 1+m^{0}\right) c=0 \\
& c\left[\left(m_{i} 1\right)\left(m^{0} i 1\right)+\left(m_{i} 1+m^{0}\right)\right]+x_{j=1 j 0=1}^{\infty n^{1} x^{0}{ }^{1}} e_{j j}^{\alpha}=0 \\
& \left(\left(m f m^{0}\right) i m_{i} m^{0}+1+m_{i} 1+m^{0}\right) c+\sum_{j=1 j^{0}=1}^{x_{i} 1 m^{0} \chi_{1}^{1}} e_{j}^{a}=0 \\
& \left(m f m^{0}\right) c+\sum_{j=1 j^{0}=1}^{n n^{0} X^{0}{ }^{1}} e_{j}^{a}=0 \\
& c=\frac{i_{1}^{P}{ }_{m_{i 1} P} m_{1}^{0}{ }_{i 1} e_{j j^{0}}^{\alpha}}{m f m^{0}}
\end{aligned}
$$

From here, we recover the coec cients (in this case dixerentials with respect to the main exects) as always,

$$
e_{j} 0=e_{j j}^{a}+c
$$

From the traditional way,

$$
a_{1 ; m}+a_{2 ; m^{0}}+c=f_{m m^{0}}
$$

where $a_{1 ; m} ; a_{2 ; m^{0}}$ are the coet cients of the main exects for two attributes and their last levels and $\mathrm{fmm}_{\mathrm{mm}}$ is the interaction exect corresponding to the last level for each attribute.

From the centering of the main exects in the traditional way we get

$$
\begin{aligned}
& a_{1 ; m}=i \frac{1}{m}{ }_{1}^{n i 1} a_{1 ; j}^{\alpha} \\
& a_{2 ; m^{0}}=i{\frac{1}{m^{0}}}_{1}^{n x^{0} i}{ }^{1} a_{2 ; j}^{\infty}{ }^{\infty}
\end{aligned}
$$

where $a_{1 ; j}^{x} ; a_{2 ; j}^{x}$ are the estimated coet cients of the main exects for two attributes and their levels. 
Then

$$
f_{m m^{0}}=i \frac{1}{m}{ }_{1}^{m i} a_{1 ; j}^{\alpha} i{\frac{1}{m^{0}}}_{1}^{m x_{i}^{0}} a_{2 ; j}^{a}+c
$$

Since $d_{m m^{0}}=f_{m m^{0}}$; if we substitute this expression coming from the traditional way to code into the restriction of the new way, we obtain,

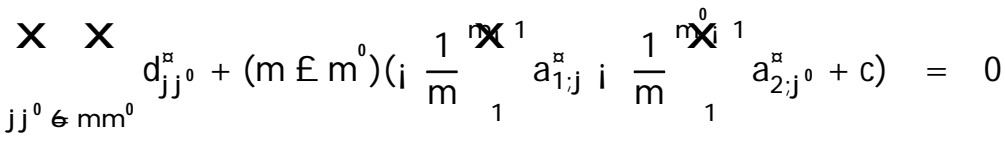

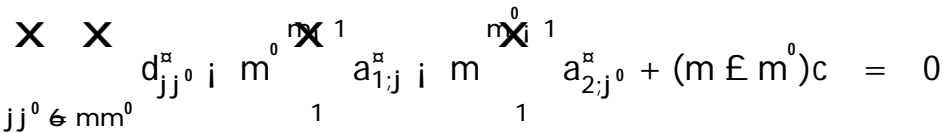

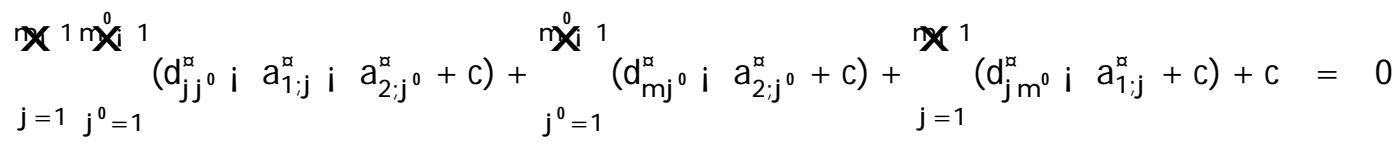

and it is equivalent to the expression used in the traditional way,

$$
{ }_{1} x_{1} 1 n^{0}{ }_{i}^{1}\left(e_{j}^{a} 0+c\right)+\left(m_{i} 1+m^{0}\right) c=0
$$

\subsection{Results}

In this particular example we have,

$$
\begin{aligned}
& \frac{.1 C C M}{\text { ICA }}=\frac{0 ; 9353}{0 ; 3117} 1 / 43 \\
& \frac{.2 C C M}{.2 C A}=\frac{0 ; 5977}{0 ; 1992} 1 / 43 \\
& \frac{.3 C C M}{\text { 3CA }}=\frac{0 ; 3234}{0 ; 1078} 1 / 43
\end{aligned}
$$

The canonical correlation coet cients for the new and the traditional way of coding the data are the following: 0:9671, 0:7731, 0:5687 and the standard coordinates for the categories:

\begin{tabular}{|cccc|}
\hline & Dim:1 & Dim:2 & Dim:3 \\
A & i $1: 238$ & 0.846 & i $0: 505$ \\
B & i $0: 426$ & i $0: 989$ & $1: 358$ \\
C & 0.518 & i $1: 343$ & i $1: 548$ \\
D & $1: 279$ & 0.879 & 0.308 \\
\hline
\end{tabular}

This agrees with the results reported in section 4.1. 


\section{Conclusions and discussions}

This paper proposed that when our objective is to analyze categorical conjoint data to obtain the utility of dixerent attribute levels, the results oxered by CCM, which is the technique normally used in this context, are the same as those oxered by the CA of a concatenated table. We have proved this idea analytically, using CC as a bridge between them, and illustrated the equivalence between the dixerent methods.

A particular applied case, a study designed by an airline company, as well as the relevant literature, suggested that to include interaction exects could be useful. We did the extension of CA and CC to be able to treat interaction exects and ..nally to CCM. The equivalence was established theoretically and illustrated with the airline data.

Given the results we can conclude that CA should be used more often in this type of analysis since the results obtained are the same as those oxered by CCM. Furthermore, CA oxers a map and this allows us to interpret the results more rapidly and easily.

As future research, it could be interesting to apply the results of this paper to more than one subject. In this new case, the matrices of dummy variables should take the form of concatenated tables with all possible combination, as before, but for all the subjects, one below the other, for the attributes as well as for the response.

A nother point to consider is what happens when we have more than one interaction exect. For the "new way" of coding, a problem appears when we want to analyze two interactions which involve one common attribute, eg. $P £ T$ and $\mathrm{P} f \mathrm{~S}$ since a double inclusion of the common attribute (in this case, price) occurs.

From an applied point of view, even though we are speaking all the time about one particular subject, from the ..rst analysis without interactions, we could get information like the fact that, for long distance travel, price is the most relevant attribute. The company to travel with is not so important if it is well known, maybe because it implies the required safety. Timetable is more valued than service, maybe because it can be perceived as quite standard between the well known airline companies. Once interaction variables are included, the information obtained can help to establish particular oxers from the travel agencies, establishing the right trade-ox between the attributes, in other words, doing optimal combinations that generate dixerent utilities than the ..rst one obtained. If individual marketing was possible to do, to this particular person, the good oxer could include, for example, the cheapest price, the company and the timetable that the travel agency prefers (except TAP), with ‡exilibity in the level of service. 


\section{R eferences}

Benzécri, J -P \& coll. (1973), L'A nalyse des Données. Tome 2: I'A nalyse des Correspondances, Dunod, Paris.

Greenacre, M.J . (1984), Theory and A pplications of Correspondance Analsyis, A cademic Press, London.

Greenacre, M.J . (1986), "SimCA : a program to perform simple correspondence analysis", American Statistician, 51, 230-231.

Greenacre, M.J. (1993), Correspondence Analysis in Practice, Academic Press, London.

Rao, V.R. (1977), Conjoint Measurement in Marketing A nalysis. In Multivariate M ethods for M arket and Survey R esearch, (ed. J agdish Sheth), University of Illinois.

Carroll, J.D., Categorical Conjoint Measurement, paper presented at the Mathematical psychology M eeting, A nn A rbor, Michigan, A ugust 1969.

Green, P.E. and Srinivasan,V. (1990), Conjoint analysis in marketing: new developments with implications for research and practice, J ournal of Marketing, 54, 3-19.

Green, P.E. and Wind, Y., Recent approaches to the modeling of individuals' subjective evaluations, paper presented at the attitude research conference, Madrid, 1972.

Green, P.E. and W ind, Y . (1973), Multiattribute Decidions in Marketing: a $M$ easurement A pproach. The Dryden Press, Hinsdale, Illinois.

Green, P.E. (1973), On the A nalysis of Interactions in Marketing Research Data, J ournal of M arketing Research, Vol X, 410-20.

Hensher, D.A. and Louviere, J. (1982), Identifying individual preferences for international air travel: An application of functional measurement theory, J ournal of Transport E conomics and Policy, 17, 225-245.

Huber,G.P. (1974), Multiattribute utility models: a review of ..led and ..eldlike studies, Management Science, 20, 1393-1402.

W ittink,D.R., Huber, J ., Zandan, P. and J ohnson, R. M . (1992), T he number of levels exect in conjoint: where does it come from, and can it be eliminated?, Sawtooth Software Conference Proceedings, K etchum, 355-364. 


\section{A ppendix I. Housing data.}

7.0.1 Conjoint analysis (Carroll, 1968). Data matrix

\begin{tabular}{|c|c|c|c|c|}
\hline House no.(M) & SIZE $\left(\mathrm{J}_{1}\right)$ & $\operatorname{PRICE}\left(\mathrm{J}_{2}\right)$ & CONDITION(J 3$)$ & TASK (J 0$)$ \\
\hline 1 & 2 & 25 & $\mathrm{E}$ & $\mathrm{A}$ \\
\hline 2 & 2 & 25 & G & A \\
\hline 3 & 2 & 25 & $P$ & C \\
\hline 4 & 2 & 30 & $E$ & A \\
\hline 5 & 2 & 30 & G & B \\
\hline 6 & 2 & 30 & $P$ & C \\
\hline 7 & 2 & 35 & $E$ & B \\
\hline 8 & 2 & 35 & $\mathrm{G}$ & B \\
\hline 9 & 2 & 35 & $P$ & D \\
\hline 10 & 2 & 40 & $E$ & B \\
\hline 11 & 2 & 40 & G & C \\
\hline 12 & 2 & 40 & $P$ & D \\
\hline 13 & 3 & 25 & $E$ & $A$ \\
\hline 14 & 3 & 25 & G & $A$ \\
\hline 15 & 3 & 25 & $P$ & C \\
\hline 16 & 3 & 30 & $E$ & $A$ \\
\hline 17 & 3 & 30 & G & B \\
\hline 18 & 3 & 30 & $P$ & C \\
\hline 19 & 3 & 35 & $E$ & B \\
\hline 20 & 3 & 35 & G & B \\
\hline 21 & 3 & 35 & $P$ & D \\
\hline 22 & 3 & 40 & $E$ & B \\
\hline 23 & 3 & 40 & G & C \\
\hline 24 & 3 & 40 & $P$ & D \\
\hline 25 & 4 & 25 & $E$ & A \\
\hline 26 & 4 & 25 & G & A \\
\hline 27 & 4 & 25 & $P$ & C \\
\hline 28 & 4 & 30 & $E$ & A \\
\hline 29 & 4 & 30 & G & B \\
\hline
\end{tabular}


7.0.2 Results

(a) Category Values

\begin{tabular}{|cccc|}
\hline SOLUTION & 1 & 2 & 3 \\
EIGENVALUES & $0 ; 9501$ & $0 ; 5166$ & $0 ; 0880$ \\
A & i $0 ; 2091$ & i $0 ; 1315$ & i $0 ; 1494$ \\
B & i $0 ; 0557$ & $0 ; 2172$ & $0 ; 0728$ \\
C & $0 ; 0923$ & i $0 ; 1747$ & $0 ; 2104$ \\
D & $0 ; 2865$ & $0 ; 0250$ & i $0 ; 2370$ \\
\hline
\end{tabular}

(b) Attribute Functions by Solution

\begin{tabular}{|c|c|c|c|c|}
\hline & & SOLUTION & NUMBER & \\
\hline ATTRIBUTE & LABEL & 1 & 2 & 3 \\
\hline \multirow[t]{3}{*}{ 1: Size of house } & $\mathrm{S} 2$ & 0 & 0 & 0 \\
\hline & S3 & 0 & 0 & 0 \\
\hline & S4 & 0 & 0 & 0 \\
\hline \multirow[t]{4}{*}{ 2:A sking Price } & $P 1=25$ & i 0:1086 & i 0:1459 & i 0:0295 \\
\hline & $P 2=30$ & ¡ 0:0575 & i 0:0297 & 0:0446 \\
\hline & $P 3=35$ & 0:0584 & 0:1531 & i 0:0305 \\
\hline & $P 4=40$ & 0:1077 & 0:0225 & 0:0154 \\
\hline \multirow[t]{3}{*}{ 3: Condition } & CE & i 0:1324 & 0:0428 & i 0:0383 \\
\hline & CG & i 0:0570 & 0:0320 & 0:0563 \\
\hline & CP & 0:1894 & i 0:0748 & ¡ 0:0133 \\
\hline
\end{tabular}

\subsection{Canonical correlation}

\subsubsection{Results}

\section{Categories (Y)}

\begin{tabular}{|cccc|}
\hline & ${\text { Canonical weights } 1^{\text {st }} \text { axis }}$ & Canonical weights $2^{\text {nd }}$ axis & Canonical weights $3^{\text {rd }}$ axis \\
A & 2.9320 & -0.9256 & 0.5180 \\
B & 2.0246 & 1.1368 & 1.8327 \\
C & 1.1488 & -1.1817 & 2.6469 \\
\hline
\end{tabular}

Attributes (X) 


\begin{tabular}{|cccc|}
\hline & Canonical weights $1^{\text {st }}$ axis & Canonical weights $2^{\text {nd }}$ axis & Canonical weights $3^{\text {rd }}$ axis \\
S2 & 0 & 0 & 0 \\
s3 & 0 & 0 & 0 \\
p1 & 1.3124 & -1.3858 & -0.8953 \\
P2 & 1.0022 & -0.4293 & 0.5822 \\
P3 & 0.2994 & 1.0752 & -0.9152 \\
CE & 1.9524 & 0.9689 & -0.4994 \\
CG & 1.4952 & 0.8799 & 1.2952 \\
\hline
\end{tabular}

Canonical correlations: $0.9752 \quad 0.7188 \quad 0.2966$

\subsection{Correspondence analysis}

Concadenated table

$\begin{array}{lllll} & \text { A } & \text { B } & \text { C } & \text { D } \\ \text { s2 } & 3 & 4 & 3 & 2 \\ \text { s3 } & 3 & 4 & 3 & 2 \\ \text { s4 } & 3 & 4 & 3 & 2 \\ \text { p1 } & 6 & 0 & 3 & 0 \\ \text { P2 } & 3 & 3 & 3 & 0 \\ \text { p3 } & 0 & 6 & 0 & 3 \\ \text { p4 } & 0 & 3 & 3 & 3 \\ \text { CE } & 6 & 6 & 0 & 0 \\ \text { CG } & 3 & 6 & 3 & 0 \\ \text { CP } & 0 & 0 & 6 & 6\end{array}$

Inertia and percentages of inertia

\begin{tabular}{lll}
1 & 0.4755 & $61.14 \% * * * * * * * * * * * * * * * * * * * * * * * * * * * * * * * *$ \\
2 & 0.2583 & 33. $21 \% * * * * * * * * * * * * * * * * * * * * * * * * * * * *$ \\
3 & 0.0440 & $5.65 \% * * * * *$ \\
\hdashline .2778 &
\end{tabular}

\section{R ow Contributions}

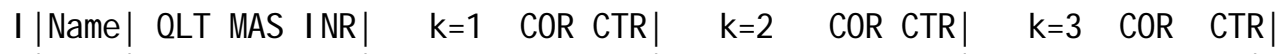

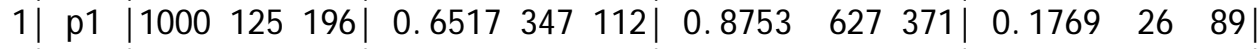

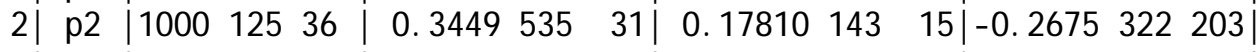

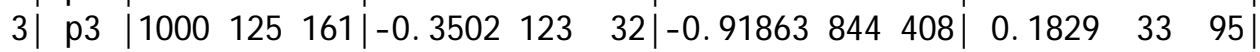
4| p4 | \begin{tabular}{llllllll|lll|}
1000 & 125 & 71 & $\mid-0.6463$ & 940 & $110 \mid-0.13482$ & 41 & $9 \mid$ & -0.0923 & 19 & $24 \mid$
\end{tabular}

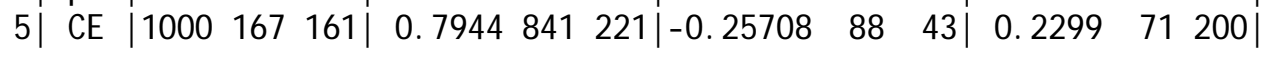


6| CG | 100016754 | $0.3422468 \quad 41|-0.19216148 \quad 24|-0.3098384364 \mid$

7| $\quad$ CP | $1000167321|-1.1366861453| \begin{array}{llllllll}13 & 0.44924 & 135 & 130 \mid & 0.0798 & 4 & 24\end{array}$

The attribute service does not appear given that its contribution is zero.

Column Contributions

Nanæ| QT MAS I NR $\quad k=1 \quad C O R$ CTR $\quad k=2 \quad C O R$ CTR $\quad k=3 \quad$ COR CTR

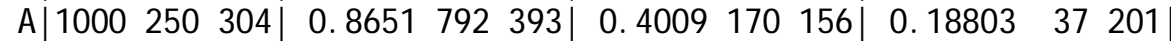

B| $1000333214 \mid$\begin{tabular}{lllllll|ll|l}
0.2304 & 106 & $37 \mid-0.6622$ & 877 & $566 \mid-0.09157$ & 17 & $64 \mid$
\end{tabular}

C $1000250161|-0.3820292 \quad 77| \begin{array}{lllllll}0.5329 & 568 & 275 \mid-0.26474 & 140 & 398 \mid\end{array}$

D| $1000167321|-1.1854937493|-0.0762 \quad 4 \quad 4 \mid \begin{array}{lllll}0.29820 & 59 & 337 \mid\end{array}$

\section{APPENDIX II. AIRLINE DATA}

\subsection{Correspondence analysis.}

\subsubsection{Data M atrix}

\begin{tabular}{|c|c|c|c|}
\hline & $A$ & $B$ & C \\
\hline $\mathrm{PT}_{11}$ & 12 & 3 & 0 \\
\hline $\mathrm{PT}_{12}$ & 12 & 3 & 0 \\
\hline $\mathrm{PT}_{13}$ & 12 & 3 & 0 \\
\hline $\mathrm{PT}_{21}$ & 0 & 12 & 3 \\
\hline $\mathrm{PT}_{22}$ & 12 & 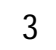 & 0 \\
\hline $\mathrm{PT}_{23}$ & 12 & 3 & 0 \\
\hline $\mathrm{PT}_{31}$ & 0 & 0 & 14 \\
\hline $\mathrm{PT}_{32}$ & 0 & 9 & 5 \\
\hline $\mathrm{PT}_{33}$ & 4 & 10 & 1 \\
\hline $\mathrm{PT}_{41}$ & 0 & 0 & 0 \\
\hline $\mathrm{PT}_{42}$ & 0 & 0 & 7 \\
\hline $\mathrm{PT}_{43}$ & 0 & 10 & 4 \\
\hline $\mathrm{PT}_{51}$ & 0 & 0 & 0 \\
\hline $\mathrm{PT}_{52}$ & 0 & 0 & 0 \\
\hline $\mathrm{PT}_{53}$ & 0 & 0 & 7 \\
\hline TWA & 16 & 10 & 8 \\
\hline IBERIA & 16 & 8 & 6 \\
\hline KLM & 16 & 10 & 8 \\
\hline B.A. & 16 & 10 & 9 \\
\hline TAP & 0 & 18 & 10 \\
\hline $\mathrm{S}_{1}$ & 20 & 16 & 12 \\
\hline $\mathrm{S}_{2}$ & 20 & 21 & 15 \\
\hline $\mathrm{S}_{3}$ & 24 & 19 & 14 \\
\hline
\end{tabular}

The previous attributes, price and timetable and their levels, appear in the analysis as supplementary points. 


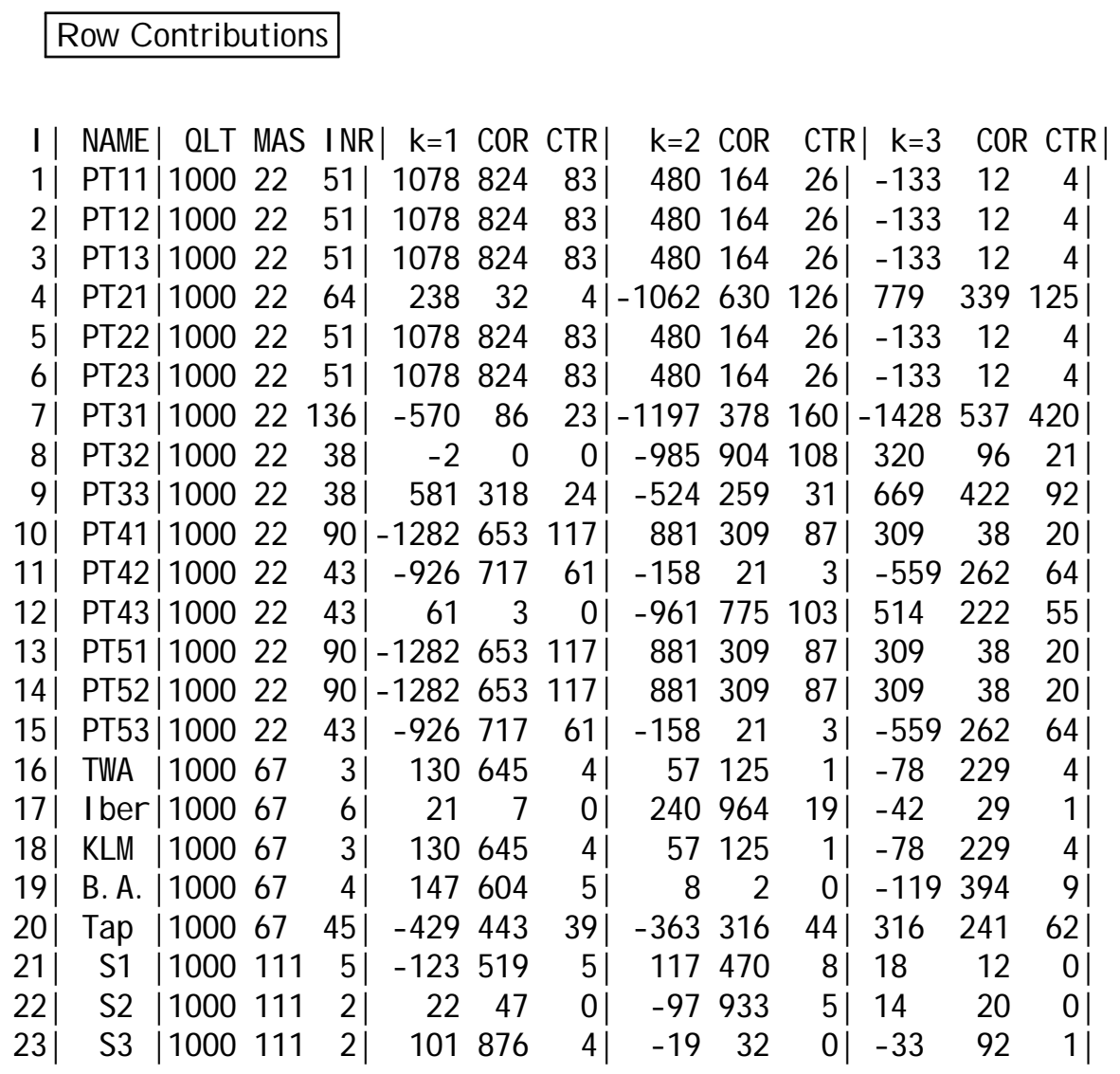

Suppl enent ary poi nts

IS| NAME| QLT MAS INR $k=1$ COR CTR $k=2$ COR CTR| $k=3$ COR CTR

24| $\quad \mathrm{P1} \mid$\begin{tabular}{lllllllll|lll|}
1000 & 67 & 152 & 1078 & 824 & 249 & 480 & 164 & $77 \mid$ & -133 & 12 & 11
\end{tabular}

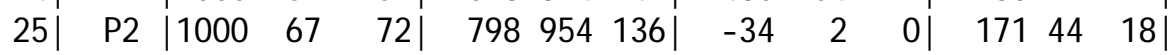

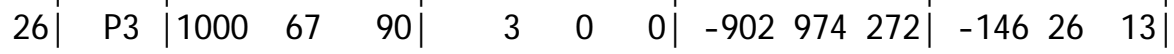

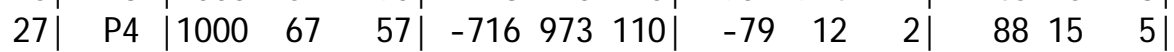

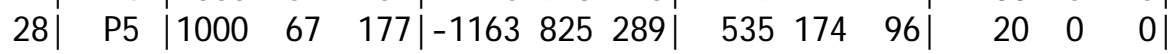

29| T1 | \begin{tabular}{lll|lll|llllll|}
1000 & 111 & $24 \mid$ & -364 & 992 & $47 \mid$ & -3 & 0 & $0 \mid$ & -33 & 8 & $1 \mid$
\end{tabular}

30| T2 | \begin{tabular}{llllll|llllll}
1000 & 111 & $4 \mid$ & -11 & 6 & $0 \mid$ & 140 & 922 & $11 \mid$ & -39 & 72 & 2
\end{tabular}

31| T3 | 1000 111 29| $374 \begin{array}{llllllll}1055 & 50 \mid & -137 & 114 & 10 \mid & 72 & 31 & 5\end{array}$

\section{Column contributions}

J) NAME| QLT MAS I NR $k=1$ COR CTR $k=2$ COR CTR $\quad k=3$ COR CTR

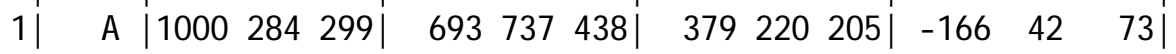

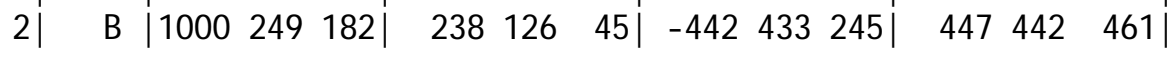




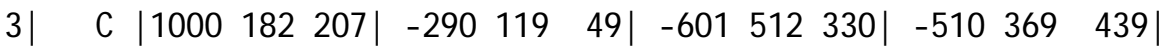

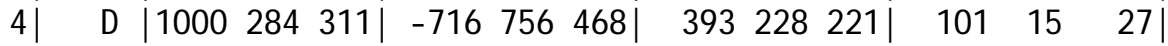

\subsection{Canonical Correlation. New way of coding.}

Linear combinations for ..rst canonical correlation. Number of obsservations = 225.

Response categories,

$\begin{array}{ccccc} & \text { Coef. } & \text { Std. Error } & t & \text { p }>\text { jtj } \\ \text { A } & \text { i } 2: 5174 & : 04683 & \text { i } 53: 756 & 0: 000 \\ \text { B } & \text { i } 1: 7052 & : 04847 & \text { i } 35: 179 & 0: 000 \\ \text { C } & \text { i }: 7614 & : 05230 & \text { i } 14: 369 & 0: 000\end{array}$

Attribute levels,

\begin{tabular}{|c|c|c|c|c|}
\hline TWA & $\begin{array}{l}\text { Coef. } \\
\text { i : :5770 }\end{array}$ & $\begin{array}{c}\text { Std. Error } \\
: 0581\end{array}$ & $\begin{array}{c}t \\
\text { 9:928 }\end{array}$ & $\begin{array}{l}p>j t j \\
0: 000\end{array}$ \\
\hline IBERIA & i :4637 & :0581 & i 7:977 & $0: 000$ \\
\hline KLM & i :5771 & :0581 & ; 9:928 & $0: 000$ \\
\hline B.A. & i :5946 & :0581 & i 10:229 & $0: 000$ \\
\hline $\mathrm{S}_{1}$ & 2303 & :0450 & 5:116 & $0: 000$ \\
\hline $\mathrm{S}_{2}$ & :0813 & :0450 & 1:806 & $0: 072$ \\
\hline $\mathrm{PT}_{11}$ & i 2:0677 & 1007 & i 20:537 & $0: 000$ \\
\hline $\mathrm{PT}_{12}$ & i 2:0677 & :1007 & i 20:537 & $0: 000$ \\
\hline $\mathrm{PT}_{13}$ & i 2:0677 & 1007 & i $20: 537$ & $0: 000$ \\
\hline $\mathrm{PT}_{21}$ & i $1: 2007$ & 1007 & i $11: 925$ & $0: 000$ \\
\hline $\mathrm{PT}_{22}$ & i 2:0677 & 1007 & i $20: 537$ & $0: 000$ \\
\hline $\mathrm{PT}_{23}$ & і 2:0677 & 1007 & i $20: 537$ & $0: 000$ \\
\hline $\mathrm{PT}_{31}$ & i : :3674 & 1007 & i 3:649 & 0:000 \\
\hline $\mathrm{PT}_{32}$ & ¡ :9530 & 1007 & i 9:465 & $0: 000$ \\
\hline $\mathrm{PT}_{33}$ & i 1:5547 & 1007 & i $15: 442$ & $0: 000$ \\
\hline $\mathrm{PT}_{41}$ & :3674 & 1007 & $3: 649$ & $0: 000$ \\
\hline $\mathrm{PT}_{42}$ & 0 & 1007 & 0:000 & $1: 000$ \\
\hline $\mathrm{T}_{43}$ & i 1:0180 & 1007 & i $10: 112$ & 0:000 \\
\hline b1 & :3674 & 1007 & $3: 649$ & 0:000 \\
\hline & :3674 & 1007 & $3: 649$ & 0.000 \\
\hline
\end{tabular}

Canoni cal correl ati ons:

0.96710 .77310 .5687

We recover the missing coę cients by centering:

Companies:

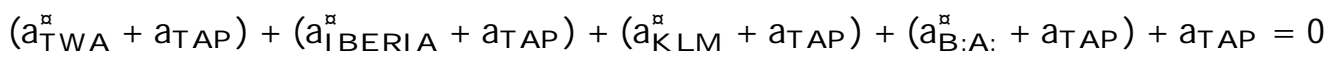




$$
\begin{aligned}
\text { aTAP } & =0: 443 \\
\text { aTWA }_{\text {T }} & i 0: 134 \\
a_{\text {IERRIA }} & i 0: 021 \\
a_{K L M} & =i 0: 134 \\
a_{B: A}: & =i 0: 152
\end{aligned}
$$

Services:

$$
\begin{aligned}
\left(a_{\mathrm{s}_{1}}^{\mathrm{a}}+a_{\mathrm{s}_{3}}\right)+\left(a_{\mathrm{s}_{2}}^{\mathrm{a}}+a_{\mathrm{s}_{3}}\right)+a_{\mathrm{s}_{3}}=0 & \\
a_{\mathrm{s}_{1}} & =0: 126 \\
a_{\mathrm{s}_{2}} & =\mathrm{i} 0: 023 \\
a_{\mathrm{s}_{3}} & =\mathrm{i} 0: 104
\end{aligned}
$$

Interaction variable PT:

$$
\begin{gathered}
\left(a_{P T_{11}}^{\infty}+a_{P T_{53}}\right)+\left(a_{P T_{12}}^{\infty}+a_{p T_{53}}\right)+:::+a_{P T_{53}}=0 \\
a_{p T_{53}}=0: 955
\end{gathered}
$$

We recover the true values:

$$
\begin{array}{lllll}
\mathrm{apT}_{11}=\mathrm{i} 1: 113 & \mathrm{apT}_{21}=\mathrm{i} 0: 245 & \mathrm{apT}_{31}=0: 588 & \mathrm{apT}_{41}=1: 322 & \mathrm{apT}_{51}=1: 322 \\
\mathrm{apT}_{12}=\mathrm{i} 1: 113 & \mathrm{ap}_{22}=\mathrm{i} 1: 113 & \mathrm{apT}_{32}=0: 002 & \mathrm{apT}_{42}=0: 955 & \mathrm{apT}_{52}=1: 322 \\
\mathrm{apT}_{13}=\mathrm{i} 1: 113 & \mathrm{apT}_{23}=\mathrm{i} 1: 113 & \mathrm{apT}_{33}=\mathrm{i} 0: 595 & \mathrm{apT}_{43}=\mathrm{i} 0: 063 & \mathrm{apT}_{53}=0: 955
\end{array}
$$

\subsection{Canonical Correlation. Traditional way of coding.}

Linear combinations for ..rst canonical correlation. Number of observations = 225.

Response categories,

$\begin{array}{ccccc} & \text { Coef. } & \text { Std. Error } & t & \text { p }>\text { jtj } \\ \text { A } & \text { i } 2: 5174 & : 04683 & \text { i } 53: 756 & 0: 000 \\ \text { B } & \text { i } 1: 7052 & : 04847 & \text { i } 35: 179 & 0: 000 \\ \text { C } & \text { i }: 7614 & : 05230 & \text { i } 14: 369 & 0: 000\end{array}$


Attribute levels,

\begin{tabular}{|c|c|c|c|c|}
\hline & Coef: & Std:Error & t & $P>j t j$ \\
\hline$P_{1}$ & 2:0677 & :10068 & $20: 537$ & 0:000 \\
\hline$P_{2}$ & 2:0677 & :10068 & $20: 537$ & 0:000 \\
\hline$P_{3}$ & 1:5547 & 10068 & $15: 442$ & 0:000 \\
\hline$P_{4}$ & 1:0180 & :10068 & $10: 112$ & 0:000 \\
\hline TWA & 5771 & :05813 & 9:928 & 0:000 \\
\hline IBERIA & :4637 & :05813 & 7:977 & 0:000 \\
\hline KLM & :5771 & :05813 & 9:928 & 0:000 \\
\hline B.A. & :5946 & :05813 & $10: 229$ & 0:000 \\
\hline $\mathrm{T}_{1}$ & i : :3674 & :10068 & і 3:649 & 0:000 \\
\hline $\mathrm{T}_{2}$ & i : 3674 & 10068 & i 3:649 & 0:000 \\
\hline $\mathrm{S}_{1}$ & i : 2305 & :04503 & i 5:116 & 0:000 \\
\hline $\mathrm{S}_{2}$ & i : 0813 & :04503 & i 1:806 & 0:072 \\
\hline $\mathrm{PT}_{11}$ & :3674 & 14238 & 2:581 & 0:011 \\
\hline $\mathrm{PT}_{12}$ & :3674 & :14238 & 2:581 & 0:011 \\
\hline $\mathrm{PT}_{21}$ & i : 5000 & :14238 & ; 3:509 & 0:001 \\
\hline $\mathrm{PT}_{22}$ & :3674 & 14238 & 2:581 & 0:011 \\
\hline $\mathrm{PT}_{31}$ & i :8199 & 14238 & i $5: 758$ & 0:000 \\
\hline $\mathrm{PT}_{32}$ & ¡ : 2343 & 14238 & i 1:646 & 0:101 \\
\hline $\mathrm{PT}_{41}$ & ¡ 1:0180 & 14238 & i $7: 150$ & 0:000 \\
\hline $\mathrm{PT}_{42}$ & i :6506 & 14238 & ; 4:569 & 0:000 \\
\hline
\end{tabular}

Canonical correlations: $0.9671 \quad 0.7731 \quad 0.5687$

From the results we corroborate that the canonical correlation coec cients are the same if we code the data in the new way and if we do it in the traditional form. Further, the coec cients for the main exects are also the same.

The remaining work is to ..nd the values for the interactions in the new form.

The restriction to be applied in this case is the following:

$$
\begin{gathered}
\left(a_{P_{T_{11}}}^{0}+c\right)+\left(a_{P_{12}}^{0}+c\right)+c+\left(a_{P_{21}}^{0}+c\right)+\left(a_{P_{T_{22}}}^{0}+c\right)+c+:::+3 c=0 \\
c=0: 141
\end{gathered}
$$

Then, the true coec cients are recovered in the following way:

$$
\begin{aligned}
& a_{\mathrm{PT}_{11}}=a_{\mathrm{P}_{1}}+a_{T_{1}}+\left(a_{\mathrm{pT}_{11}}^{0}+c\right) \\
& \mathrm{ap}_{11}=1: 113
\end{aligned}
$$

which dixer from the previous recovered values only in their sign. The operation is repeated for all the other coec cients obtaining the following solutions:

$$
\begin{aligned}
& a_{\mathrm{pt}_{21}}=0: 245 \quad a_{\mathrm{pT}_{31}}=\mathrm{i} 0: 588 \quad \mathrm{apT}_{41}=\mathrm{i} 1: 322 \quad \mathrm{apt}_{51}=\mathrm{i} 1: 322 \\
& a_{\mathrm{pt}_{22}}=1: 113 \quad \mathrm{apT}_{32}=\mathrm{i} \text { 0:002 } \quad \mathrm{apT}_{42}=\mathrm{i} \text { 0:955 } \quad \mathrm{apT}_{52}=\mathrm{i} 1: 322 \\
& a_{\mathrm{p}_{23}}=1: 113 \quad a_{\mathrm{pT}_{33}}=0: 595 \quad a_{\mathrm{pT}_{43}}=0: 063 \quad a_{\mathrm{p}_{53}}=\mathrm{i} \text { 0:955 }
\end{aligned}
$$




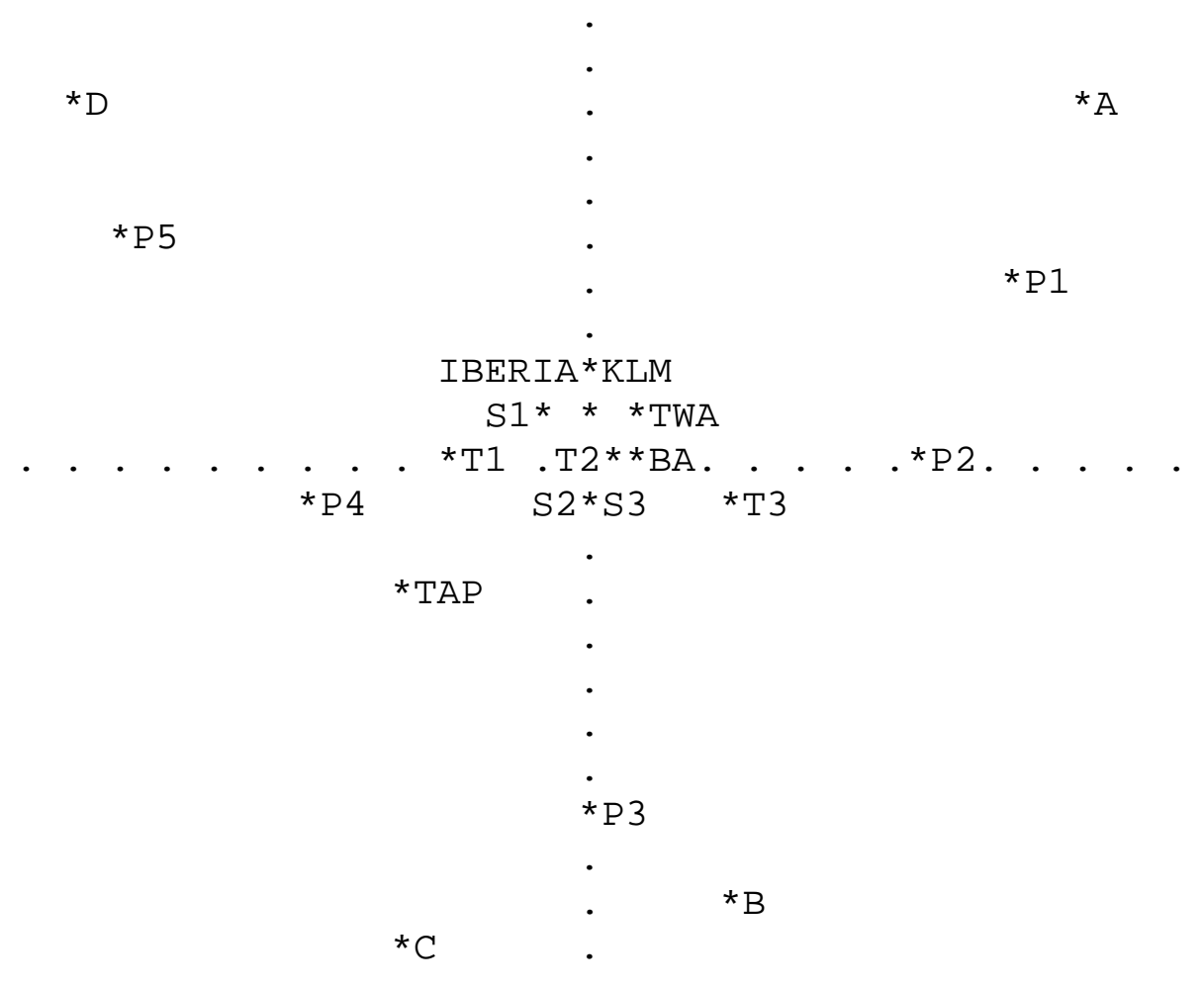

Figure 1:

Correspondence A nalysis A SCII Map by SimCA

Horizontal axis is dimension 1 with inertia $=0.2206(70.5 \%)$

Vertical axis is dimension 2 with inertia $=0.0821(26.2 \%)$

$96.7 \%$ of total inertia is represented in the above map. 


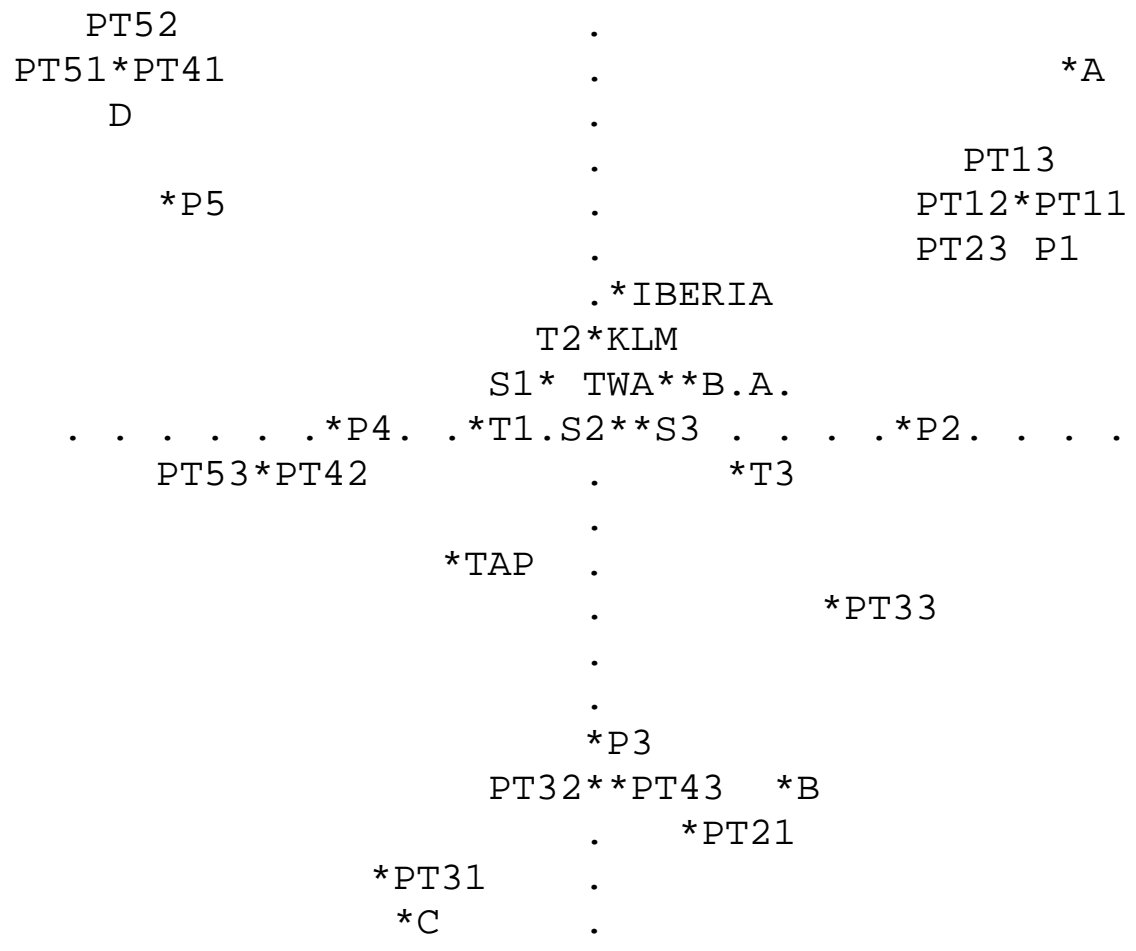

Figure 2:

Correspondence A nalysis A SCII M ap by SimCA

Horizontal axis is dimension 1 with inertia $=0.3117(50.4 \%)$

Vertical axis is dimension 2 with inertia $=0.1992(32.2 \%)$

$82.6 \%$ of total inertia is represented in the above map 